\title{
Abundant Explicit and Exact Solutions for the Variable Coefficient mKdV Equations
}

\author{
Xiaoxiao Zheng, ${ }^{1}$ Yadong Shang, ${ }^{1,2}$ and Yong Huang ${ }^{3}$ \\ ${ }^{1}$ School of Mathematics and Information Science, Guangzhou University, Guangzhou, Guangdong 510006, China \\ ${ }^{2}$ Key Laboratory of Mathematics and Interdisciplinary Sciences of Guangdong, Higher Education Institutes, \\ Guangzhou University, Guangzhou, Guangdong 510006, China \\ ${ }^{3}$ School of Computer Science and Educational Software, Guangzhou University, Guangzhou, Guangdong 510006, China
}

Correspondence should be addressed to Yadong Shang; gzydshang@126.com

Received 30 October 2013; Accepted 7 December 2013

Academic Editor: Dianchen Lu

Copyright (C) 2013 Xiaoxiao Zheng et al. This is an open access article distributed under the Creative Commons Attribution License, which permits unrestricted use, distribution, and reproduction in any medium, provided the original work is properly cited.

This paper is concerned with the variable coefficients $\mathrm{mKdV}(\mathrm{VC}-\mathrm{mKdV})$ equation. First, through some transformation we convert VC-mKdV equation into the constant coefficient $\mathrm{mKdV}$ equation. Then, using the first integral method we obtain the exact solutions of VC-mKdV equation, such as rational function solutions, periodic wave solutions of triangle function, bell-shape solitary wave solution, kink-shape solitary wave solution, Jacobi elliptic function solutions, and Weierstrass elliptic function solution. Furthermore, with the aid of Mathematica, the extended hyperbolic functions method is used to establish abundant exact explicit solution of VC-mKdV equation. By the results of the equation, the first integral method and the extended hyperbolic function method are extended from the constant coefficient nonlinear evolution equations to the variable coefficients nonlinear partial differential equation.

\section{Introduction}

It is well known that the $\mathrm{KdV}$ equation plays an important role in the soliton theory. Many properties of the KdV equation, such as symmetry, Bäcklund transformation, infinite conservation laws, Lax pairs, and Painleve analysis, have been studied. Miura transformation links the KdV equation with the $\mathrm{mKdV}$ equation. Therefore, as the $\mathrm{KdV}$ equation, $\mathrm{mKdV}$ equation is also important in mathematical physics field. In recent years, some authors considered the constant coefficients $\mathrm{mKdV}$ equation [1-4]. However, in practical applications, the coefficients of nonlinear evolution equations vary with time and space. Therefore, the exact solution of the variable coefficient nonlinear evolution equations has a greater application value.

This paper will discuss the variable coefficients $\mathrm{mKdV}$ equation (VC-mKdV):

$$
u_{t}+a(t) u_{x}+b(t) u^{2} u_{x}+r(t) u_{x x x}=0,
$$

where $a(t), b(t)$, and $r(t)$ are arbitrary function of variable $t$. More recently, some properties of the variable coefficients mKdV equation have been studied [5-18]. The aim of this paper is to apply the first integral method and the extended hyperbolic function method for constructing a series of explicit exact solutions to the VC-mKdV equation (1), such as rational function solutions, periodic wave solutions of triangle functions, bell-shape solitary wave solution, kink-shape solitary wave solution, Jacobi elliptic function solutions, Weierstrass elliptic function solution, and many exact explicit solutions in form of the rational function of hyperbolic function and the rational function of triangle function.

The rest of this paper is organized as follows. In Section 2, the outline of the first integral method will be given. In Section 3 we introduce a transformation to transform the VC-mKdV equation (1) into a constant coefficients $\mathrm{mKdV}$ equation. Section 4 is the main part of this paper; the methods are employed to seek the explicit and exact solutions 
of the VC-mKdV equation (1). In the last section, some conclusion is given.

\section{The First Integral Method}

The first-integral method, which is based on the ring theory of commutative algebra, was first proposed by Professor Feng Zhaosheng [19] in 2002. The method has been applied by Feng to solve Burgers-KdV equation, the compound Burgers$\mathrm{KdV}$ equation, an approximate Sine-Gordon equation in $(n+$ $1)$-dimensional space, and two-dimensional Burgers-KdV equation [20-24].

In the recent years, many authors employed this method to solve different types of nonlinear partial differential equations in physical mathematics. More information about these applications can be found in [25] and references therein. The most advantage is that the first integral method has not many sophisticated computation in solving nonlinear algebra equations compared to other direct algebra method. For completeness, we briefly outline the main steps of this method.

The main steps of this method are summarized as follows.

Given a system of nonlinear partial differential equations, for example, in two independent variables,

$$
P\left(u_{t}, u_{x}, u_{x x}, u_{x t}, \ldots\right)=0 .
$$

Using traveling wave transformation $u(x, t)=f(\xi), \xi=k x+$ $\omega t+\xi_{0}$ and some other mathematical operations, the systems (2) can be reduced to a second-order nonlinear ordinary differential equation:

$$
D\left(f, f^{\prime}, f^{\prime \prime}\right)=0 \text {. }
$$

By introducing new variables $X=f(\xi), Y=f^{\prime}(\xi)$ or making some other transformations, we reduce ordinary differential equation (3) to a system of the first order ordinary differential equation:

$$
\begin{gathered}
X^{\prime}=Y \\
Y^{\prime}=H(X, Y) .
\end{gathered}
$$

Suppose that the first integral of (4) has a form as follows:

$$
P(X, Y)=\sum_{i=0}^{m} a_{i}(X) Y^{i}=0 .
$$

(In general $m=1$ or $m=2)$, where $a_{i}(X)(i=0,1, \ldots, m)$ are real polynomials of $X$.

According to the Division theorem from ring theory of commutative algebra, there exists polynomials $\alpha(X), \beta(X)$ of variable $X$ in $\mathfrak{R}[X]$ such that

$$
\frac{d P}{d \xi}=[\alpha(X)+\beta(X) Y] P(X, Y) .
$$

We determine polynomials $\alpha(X), \beta(X), a_{i}(X)(i=0,1$, $2, \ldots$ ) from (6), furthermore, obtain $P(X, Y)$.

Then substituting $X=f(\xi), Y=f^{\prime}(\xi)$ or other transformations into (5), exact solutions to (2) are established, through solving the resulting first-order integrable differential equation.

\section{A Transformation to the VC-mKdV Equation}

In order to transfer (1) into the form of (3), we firstly do some transformations for (1). Since (1) is a variable coefficients equation and we need to transform it to the constant coefficients $\mathrm{mKdV}$ equation, we introduce a transformation

$$
u(x, t)=U(X, T) p(x, t)
$$

where $X=X(x, t), T=T(x, t)$. Through this transformation, we hope that (1) be changed into the form of constant coefficients $\mathrm{mKdV}$ equation:

$$
U_{T}+24 U^{2} U_{X}+U_{X X X}=0 .
$$

In order to obtain the above transformation equation, substituting (7) into (1) and assuming $T_{x}=0$, and simultaneously on both side of the formulas by dividing $r(t) X_{x}^{3}$, we have

$$
\begin{gathered}
\frac{p b U^{3} p_{x}}{r X_{x}^{3}}+\frac{p^{2} b U^{2} U_{X}}{r X_{x}^{2}}+\left(\frac{p_{t}}{r X_{x}^{3} p}+\frac{p_{x x x}}{X_{x}^{3} p}+\frac{a p_{x}}{r X_{x}^{3} p}\right) U \\
+\left(\frac{a}{r X_{x}^{2}}+\frac{X_{t}}{r X_{x}^{3}}+\frac{X_{x x x}}{X_{x}^{3}}+\frac{2 p_{x x}}{X_{x}^{2} p}+\frac{3 X_{x x} p_{x}}{X_{x}^{3} p}\right) U_{X} \\
+\frac{T_{t}}{r X_{x}^{3}} U_{T}+\left(\frac{3 X_{x x}}{X_{x}^{2}}+\frac{3 p_{x}}{X_{x} p}\right) U_{X X}+U_{X X X}=0
\end{gathered}
$$

Comparing the coefficients of (9) with (8), such as $U^{2} U_{X}$, $U, U_{x}$, and so on, we have

$$
\begin{gathered}
\frac{p b p_{x}}{r X_{x}^{3}}=0, \\
\frac{p^{2} b}{r X_{x}^{2}}=24 \\
\frac{p_{t}}{r X_{x}^{3} p}+\frac{p_{x x x}}{X_{x}^{3} p}+\frac{a p_{x}}{r X_{x}^{3} p}=0, \\
\frac{X_{t}}{r X_{x}^{3}}+\frac{X_{x x x}}{X_{x}^{3}}+\frac{2 p_{x x}}{X_{x}^{2} p}+\frac{3 X_{x x} p_{x}}{X_{x}^{3} p}=0, \\
\frac{T_{t}}{r X_{x}^{3}}=1, \\
\frac{3 X_{x x}}{X_{x}^{2}}+\frac{3 p_{x}}{X_{x} p}=0 .
\end{gathered}
$$

From (11) and (14), we have $X_{x} \neq 0$ and

$$
b=\frac{24 r X_{x}^{2}}{p^{2}}, \quad r=\frac{T_{t}}{X_{x}^{3}} .
$$


Substituting (16) into (10), (12), (13), and (15), we obtain

$$
\begin{gathered}
\frac{24 p_{x}}{X_{x} p}=0 \\
\frac{X_{x}^{3} p_{t}+p_{x x x} T_{t}+X_{x}^{3} a p_{x}}{T_{t} X_{x}^{3} p}=0 \\
\frac{a X_{x}^{4} P+X_{x}^{3} X_{t} p+X_{x x x} T_{t} p+2 p_{x x} T_{t} X_{x}+3 X_{x x} p_{x} T_{t}}{T_{t} X_{x}^{3} p}=0 \\
\frac{3\left(X_{x x} p+p_{x} X_{x}\right)}{X_{x}^{2} p}=0
\end{gathered}
$$

Form (18), we have $T_{t} \neq 0$. Also from (20), we have $X_{x x}=0$ and $X_{x x x}=0$. From (17) and (18), we have that $p$ can only be a constant. For simplicity, we take $p=C$. Substituting this into (19), we obtain

$$
X=F\left(x-\int a(t) d t\right) .
$$

For simplicity, we take

$$
X=x-\int a(t) d t
$$

Substituting (23) into (16), we have

$$
T=\int r(t) d t+C_{1}
$$

where $C_{1}$ is an arbitrary constant. Thus we get $s$ transform between VC-mKdV equation (1) with constant coefficients mKdV equation (8).

\section{Explicit and Exact Solutions of the VC-mKdV Equation}

We firstly obtain explicit and exact solutions of the constant coefficients mKdV equation (8) and then obtain explicit and exact solutions of the constant coefficients $\mathrm{mKdV}$ equation (1). In the view of (8), we suppose $\xi=k X+\omega T+\xi_{0}$, and then we have

$$
\omega U^{\prime}+24 k U^{2} U^{\prime}+k^{3} U^{\prime \prime \prime}=0 .
$$

Integrating (24) once with respect to $\xi$, we obtain

$$
U^{\prime \prime}+m U^{3}+l U=E,
$$

where $l=\omega / k^{3}, m=8 / k^{2}$, and $E$ is arbitrary integration constant. Let $R=U(\xi), S=R^{\prime}$; (25) can be converted to a system of nonlinear ODEs as follows:

$$
\begin{gathered}
R^{\prime}=S, \\
S^{\prime}=-m R^{3}-l R+E .
\end{gathered}
$$

Now we employ the Division theorem to seek the first integral to (26). Suppose that $R=R(\xi), S=S(\xi)$ are the nontrivial solution to the system (26), and its first integral is an irreducible polynomial in $\mathfrak{R}[R, S]$ :

$$
P(R(\xi), S(\xi))=\sum_{i=0}^{2} a_{i}(R) S^{i}=0,
$$

where $a_{i}, i=0,1,2$ are polynomial of $R$. According to the Division theorem, there exists polynomials $\alpha(R), \beta(R)$ of variable $R$ in $\Re[R]$ such that

$$
\begin{aligned}
\frac{d P}{d \xi}= & a_{2}^{\prime}(R) S^{3}+2 a_{2}(R) S S^{\prime}+a_{1}^{\prime}(R) S^{2} \\
& +a_{1}(R) S^{\prime}+a_{0}^{\prime}(R) S \\
= & (\alpha(R)+\beta(R) S)\left(a_{2}(R) S^{2}+a_{1}(R) S+a_{0}(R)\right) .
\end{aligned}
$$

Collecting all the terms with the same power of $S$ together and equating each coefficient to zero yield a set of nonlinear algebraic equations as follows:

$$
\begin{gathered}
a_{2}^{\prime}(R)=\beta(R) a_{2}(R), \\
a_{1}^{\prime}(R)=\beta(R) a_{1}(R)+\alpha(R) a_{2}(R), \\
a_{0}^{\prime}(R)=\beta(R) a_{0}(R)+\alpha(R) a_{1}(R) \\
+2 a_{2}(R)\left(m R^{3}+l R-E\right), \\
a_{1}(R)\left(-m R^{3}-l R+E\right)=\alpha(R) a_{0}(R) .
\end{gathered}
$$

Because $a_{i}(R),(i=1,2)$ are polynomials, from (29) we can deduce $\operatorname{deg}\left[a_{2}(R)\right]=0, \beta(R)=0$; that is $a_{2}(R)$ is a constant. For simplicity, we take $\beta(R)=0, a_{2}(R)=1$. Then we determine $a_{0}(R), a_{1}(R)$, and $\alpha(R)$. From (30), we have $\operatorname{deg}\left[a_{1}(R)\right]-1=\operatorname{deg}[\alpha(R)]$ or $a_{1}(R)=\alpha(R)=0$. In what follows we will discuss these two situations.

(1) In the case of $\alpha(R)=0, a_{1}(R)=0$.

In this case, (30) and (32) are satisfied. From (31), we can derive $a_{0}(R)=(m / 2) R^{4}+l R^{2}-2 E R+d$, where $d$ is an integral constant. Substituting $a_{2}(R), a_{1}(R), a_{0}(R)$ into (27), one obtains that

$$
R^{\prime}= \pm \sqrt{-\frac{m}{2} R^{4}-l R^{2}+2 E R-d} .
$$

Based on the discussion for different parameters, we can obtain the solutions of the nonlinear ordinary differential equation (33).

(a) For $d=E=0,(33)$ admits the following three general solutions:

$$
\begin{aligned}
& R_{1}= \pm \sqrt{\frac{2 l}{m} \operatorname{csch} \sqrt{-l} \xi, \quad l m>0} \\
& R_{2}= \pm \sqrt{-\frac{2 l}{m} \operatorname{sech} \sqrt{-l} \xi, \quad l<0, m>0} \\
& R_{3}= \pm \frac{1}{\sqrt{-m / 2} \xi}, \quad l=0 .
\end{aligned}
$$


Combining (7), (22), (23), (34), and $p(x, t)=C, R=U(\xi)$, one can get the following three sets of explicit exact solutions to (1):

$$
\begin{aligned}
& u_{1}(x, t)= \pm i C \sqrt{\frac{\omega}{4 k}} \operatorname{csch} \sqrt{\frac{\omega}{k^{3}}} \xi, \quad k \omega>0, \\
& u_{2}(x, t)= \pm C \sqrt{-\frac{\omega}{4 k}} \operatorname{sech} \sqrt{-\frac{\omega}{k^{3}}} \xi, \quad k \omega<0, \\
& u_{3}(x, t)= \pm \frac{C k}{2 i\left[k\left(x-\int a(t) d t\right)+\xi_{0}\right]}, \quad \omega=0,
\end{aligned}
$$

where $\xi=k\left(x-\int a(t) d t\right)+\omega\left(\int r(t) d t+C_{1}\right)+\xi_{0}, C, C_{1}$ are arbitrary parameters, and $\xi_{0}$ is an arbitrary constant.

(b) For $E=0, d=l^{2} / 2 m$, we can obtain the following four sets of explicit exact solutions to (33)

$$
\begin{aligned}
& R_{4}= \pm \sqrt{\frac{l}{m}} \tan \sqrt{-\frac{l}{2}} \xi, \quad l m>0, \\
& R_{5}= \pm \sqrt{\frac{l}{m}} \cot \sqrt{-\frac{l}{2} \xi,} \quad \text { Im }>0, \\
& R_{6}= \pm \sqrt{-\frac{l}{m}} \tanh \sqrt{\frac{l}{2} \xi,} \quad \text { Im }<0, \\
& R_{7}= \pm \sqrt{-\frac{l}{m}} \operatorname{coth} \sqrt{\frac{l}{2}} \xi, \quad \operatorname{lm}<0 .
\end{aligned}
$$

Combining (7), (22), (23), (36), and $p(x, t)=C, R=U(\xi)$, we can get the following four explicit exact solutions of (1):

$$
\begin{array}{ll}
u_{4}(x, t)= \pm C \sqrt{\frac{\omega}{8 k}} \tan \sqrt{-\frac{\omega}{2 k^{3}}} \xi, & k \omega>0, \\
u_{5}(x, t)= \pm C \sqrt{\frac{\omega}{8 k}} \cot \sqrt{-\frac{\omega}{2 k^{3}}}, \quad k \omega>0, \\
u_{6}(x, t)= \pm C \sqrt{-\frac{\omega}{8 k}} \tanh \sqrt{\frac{\omega}{2 k^{3}}} \xi, \quad k \omega<0, \\
u_{7}(x, t)= \pm C \sqrt{-\frac{\omega}{8 k}} \operatorname{coth} \sqrt{\frac{\omega}{2 k^{3}}} \xi, \quad k \omega<0,
\end{array}
$$

where $\xi=k\left(x-\int a(t) d t\right)+\omega\left(\int r(t) d t+C_{1}\right)+\xi_{0}, C, C_{1}$ are arbitrary parameters, and $\xi_{0}$ is an arbitrary constant.

(c) For $E=l=0$, from (33) we have

$$
R^{\prime}= \pm \sqrt{-\frac{m}{2} R^{4}-d} .
$$

Let $Z=R^{2}$; (38) becomes

$$
Z^{\prime}= \pm \sqrt{-\frac{m}{2} Z^{3}-d Z}
$$

While $m<0$, the above equation possesses a Weierstrass elliptic function doubly periodic wave type solution:

$$
Z=\wp\left(\sqrt{-\frac{m}{8}} \xi,-\frac{8 d}{m}, 0\right)
$$

Combining (7), (22), (23), (40), $Z=R^{2}, p(x, t)=C$, and $R=$ $U(\xi)$, we derive that (1) admits a Weierstrass elliptic function doubly periodic wave type solution:

$$
u_{8}(x, t)= \pm C \sqrt{\wp\left( \pm \frac{1}{k i} \xi,-d k^{2}, 0\right)},
$$

where $\xi=k\left(x-\int a(t) d t\right), C, C_{1}$ are arbitrary parameters, and $\xi_{0}$ is an arbitrary constant.

(d) For $E=0, d \neq 0$, we obtain elliptic function solutions for (33) as follows:

$$
\begin{gathered}
R_{9}= \pm \sqrt{\frac{1-l}{m} \mathrm{cn} \sqrt{\frac{1-l}{2}} \xi} \\
d=\frac{l^{2}-1}{2 m} \\
R_{10}= \pm \sqrt{\frac{2}{m} \mathrm{dn} \sqrt{2+l} \xi} \\
d=-\frac{2(l+1)}{m} \\
R_{11}= \pm \sqrt{-\frac{1+l}{m} \mathrm{nc} \sqrt{\frac{l-l}{2}}} \xi \\
d=-\frac{l^{2}-1}{2 m} \\
R_{12}= \pm \sqrt{-\frac{2(1+l)}{m} \mathrm{nd} \sqrt{2+l}} \xi \\
d=-\frac{2(l+1)}{\frac{1-l^{2}}{2 m} \mathrm{sd}} \sqrt{\frac{1-l}{2}}
\end{gathered}
$$


Combining (7), (22), (23), the above result (42), and $p(x, t)=$ $C, R=U(\xi)$, we can get the following six Jacobi elliptic doubly periodic wave solutions of (1):

$$
\begin{aligned}
& u_{9}(x, t)= \pm \sqrt{\frac{k^{3}-\omega}{8 k}} \operatorname{cn} \sqrt{\frac{k^{3}-\omega}{2 k^{3}}} \xi \\
& d=\frac{\omega^{3}-k^{6}}{16 k^{4}} \\
& u_{10}(x, t)= \pm \frac{k}{2} \operatorname{dn} \sqrt{\frac{2 k^{3}+\omega}{k^{3}}} \xi \\
& d=-\frac{\omega+k^{3}}{4 k} \\
& u_{11}(x, t)= \pm \sqrt{-\frac{k^{3}+\omega}{8 k}} \mathrm{nc} \sqrt{\frac{k^{3}-\omega}{2 k^{3}}} \xi \\
& d=\frac{\omega^{2}-k^{6}}{16 k^{4}} \\
& u_{12}(x, t)= \pm \sqrt{-\frac{k^{3}+\omega}{4 k}} \text { nd } \sqrt{\frac{2 k^{3}+\omega}{k^{3}} \xi} \\
& d=-\frac{k^{3}+\omega}{4 k} \\
& u_{13}(x, t)= \pm \sqrt{\frac{k^{6}-\omega^{2}}{16 k^{4}}} \text { sd } \sqrt{\frac{\omega-k^{3}}{2 k^{3}}} \xi \\
& d=\frac{\omega^{2}-k^{6}}{16 k^{4}} \\
& u_{14}(x, t)= \pm \sqrt{\frac{\omega+k^{3}}{4 k^{3}}} \operatorname{sc} \sqrt{\frac{\omega+2 k^{3}}{k^{3}}} \xi \\
& d=-\frac{\omega+k^{3}}{4 k^{3}},
\end{aligned}
$$

where $\xi=k\left(x-\int a(t) d t\right)+\omega\left(\int r(t) d t+C_{1}\right)+\xi_{0}, C, C_{1}$ are arbitrary parameters, and $\xi_{0}$ is an arbitrary constant.

(2) In the case of $\operatorname{deg}\left[a_{1}(R)\right]-1=\operatorname{deg}[\alpha(R)]$.

In this case, we assume that $\operatorname{deg}[\alpha(R)]=k_{1}, \operatorname{deg}\left[a_{0}(R)\right]=$ $k_{2}$; then we have $\operatorname{deg}\left[a_{1}(R)\right]=k_{1}+1$. Now, by balancing the degrees of both sides of (32), we can deduce that $k_{2}=4$. By balancing the degrees of both sides of (31), we can also conclude that $k_{1}=1$ or $k_{1}=0$. If $k_{1}=0$, assuming that $\alpha(R)=A_{0}, a_{1}(R)=A_{1} R+A_{2}, a_{0}(R)=C_{4} R^{4}+C_{3} R^{3}+$ $C_{2} R^{2}+C_{1} R+C_{0}$ and substituting them into (30)-(32), by equating the coefficients of the different powers of $X$ on both sides of (30) to (32), we can get that $\alpha(R)=a_{1}(R)=0$. This is contradicting with our assumption. It indicates that $k_{1} \neq 0$. While $k_{1}=1$, assuming that $a_{0}(R)=C_{4} R^{4}+C_{3} R^{3}+$ $C_{2} R^{2}+C_{1} R+C_{0}, a_{1}=A_{2} R^{2}+A_{1} R+A_{0}, \alpha(X)=B_{1} R+B_{0}$, then substituting these representations into (30)-(32), and by equating the coefficients of the different powers of $R$ on both sides of (30) to (32), we can obtain an overdetermined system of nonlinear algebraic equations:

$$
\begin{gathered}
2 A_{2}=B_{1} \\
A_{1}=B_{0} \\
4 C_{4}-2 m=A_{2} B_{1} \\
3 C_{3}=A_{1} B_{1}+A_{2} B_{0} \\
2 C_{2}-2 l=A_{1} B_{0}+A_{0} B_{1} \\
C_{1}=A_{2} B_{0}-2 E \\
-m A_{2}=B_{1} C_{4} \\
-m A_{1}=B_{1} C_{3}+C_{4} B_{0} \\
-m A_{0}-l A_{2}=B_{1} C_{2}+B_{0} C_{3} \\
E A_{2}-l A_{1}=B_{1} C_{1}+B_{0} C_{2} \\
E A_{1}-l A_{2}=B_{1} C_{0}+B_{0} C_{1} \\
E A_{0}=B_{0} C_{0} .
\end{gathered}
$$

By analyzing all kinds of possibilities, we have the following.

(a) While $B_{0}=C_{0}=0$, it leads to a contradiction.

(b) While $B_{0} \neq 0, C_{0}=0$, it also leads to a contradiction.

(c) While $B_{0}=0, C_{0} \neq 0$, we can derive that

$$
\begin{gathered}
E=0, \quad A_{1}=C_{1}=C_{3}=0, \quad C_{4}=-\frac{m}{2}, \\
C_{2}=-l, \quad C_{0}=-\frac{l^{2}}{2 m}, \quad A_{2}^{2}=-2 m, \quad A_{0}=\frac{l}{m} A_{2} .
\end{gathered}
$$

Setting (45) in (27) yields

$$
\frac{d R}{R^{2}+(l / m)}= \pm \frac{\sqrt{-2 m}}{2} d \xi .
$$

Solving (46), we can obtain solutions $R_{4}, R_{5}, R_{6}$, and $R_{7}$ again. Consequently, we obtain explicit exact solutions $u_{4}, u_{5}, u_{6}$, and $u_{7}$ to (1). Here we will not list them one by one.

We obtain various of explicit and exact solutions of (1) by using the extended hyperbolic functions method presented in [26] by author. we can get the following explicit exact solutions to (1):

$$
R_{15}= \pm \sqrt{-\frac{2}{m}} \frac{a \sinh (\xi)+b \cosh (\xi)}{a \cosh (\xi)+b \sinh (\xi)},
$$

when $l=2$, where $a, b$, and $r$ are arbitrary constants

$$
R_{16}= \pm \sqrt{\frac{2\left(a^{2}-b^{2}\right)}{m}} \frac{1}{a \cosh (\xi)+b \sinh (\xi)},
$$


when $l=-1$, where $a, b$, and $r$ are arbitrary constants such that $\left(a^{2}-b^{2}\right) m>0$

$$
\begin{aligned}
R_{17}= & \pm \sqrt{\frac{a^{2}-b^{2}-r^{2}}{2 m}} \frac{1}{a \cosh (\xi)+b \sinh (\xi)} \\
& \pm \sqrt{-\frac{1}{2 m}} \frac{a \sinh (\xi)+b \cosh (\xi)}{a \cosh (\xi)+b \sinh (\xi)+r},
\end{aligned}
$$

when $l=1 / 2$, where $a, b$, and $r$ are arbitrary constants

$$
\begin{aligned}
R_{18}= & \pm \sqrt{\frac{a^{2}-b^{2}-r^{2}}{2 m}} \frac{1}{a \cosh (\xi)+b \sinh (\xi)} \\
& \pm \sqrt{-\frac{1}{2 m}} \frac{b \cosh (\xi)-a \sinh (\xi)}{a \cosh (\xi)+b \sinh (\xi)+r}
\end{aligned}
$$

when $l=-1 / 2$, where $a, b$, and $r$ are arbitrary constants

$$
R_{19}= \pm \sqrt{-\frac{2\left(a^{2}+b^{2}\right)}{m}} \frac{1}{a \cosh (\xi)+b \sinh (\xi)},
$$

when $l=1$, where $a, b$, and $r$ are arbitrary constants

$$
R_{20}= \pm \sqrt{-\frac{2}{m}} \frac{b \cosh (\xi)-a \sinh (\xi)}{a \cosh (\xi)+b \sinh (\xi)}
$$

when $l=-2$, where $a, b$, and $r$ are arbitrary constants.

Combining (7), (22), (23), the above result (47)-(52), and $p(x, t)=C, R=U(\xi)$, the VC-mKdV equation (1) has explicit and exact solitary wave solutions:

$$
u_{15}(x, t)= \pm \frac{k i(a \sinh (\xi)+b \cosh (\xi))}{2(a \cosh (\xi)+b \sinh (\xi))},
$$

where $k, \omega, a$, and $b$ are arbitrary constants such that $\omega=2 k^{3}$, $\xi=k\left(x-\int a(t) d t\right)+\omega\left(\int r(t) d t+C_{1}\right)+\xi_{0}$

$$
u_{16}(x, t)= \pm \frac{k \sqrt{a^{2}-b^{2}}}{2} \frac{1}{a \cosh (\xi)+b \sinh (\xi)},
$$

where $k, \omega, a$, and $b$ are arbitrary constants such that $\omega=-k^{3}$, $\xi=k\left(x-\int a(t) d t\right)+\omega\left(\int r(t) d t+C_{1}\right)+\xi_{0}$

$$
\begin{aligned}
u_{17}(x, t)= & \pm \frac{k \sqrt{a^{2}-b^{2}-r^{2}}}{4} \frac{1}{a \cosh (\xi)+b \sinh (\xi)} \\
& \pm \sqrt{-\frac{1}{2 m}} \frac{a \sinh (\xi)+b \cosh (\xi)}{a \cosh (\xi)+b \sinh (\xi)+r},
\end{aligned}
$$

where $k, \omega, a$, and $b$ are arbitrary constants such that $2 \omega=k^{3}$, $\xi=k\left(x-\int a(t) d t\right)+\omega\left(\int r(t) d t+C_{1}\right)+\xi_{0}$

$$
\begin{aligned}
u_{18}(x, t)= & \pm \frac{k \sqrt{a^{2}-b^{2}-r^{2}}}{2(a \cosh (\xi)+b \sinh (\xi))} \\
& \pm \sqrt{-\frac{1}{2 m}} \frac{b \cosh (\xi)-a \sinh (\xi)}{a \cosh (\xi)+b \sinh (\xi)+r},
\end{aligned}
$$

where $k, \omega, a$, and $b$ are arbitrary constants such that $-2 \omega=$ $k^{3}, \xi=k\left(x-\int a(t) d t\right)+\omega\left(\int r(t) d t+C_{1}\right)+\xi_{0}$

$$
u_{19}(x, t)= \pm \sqrt{-\frac{2\left(a^{2}+b^{2}\right)}{m}} \frac{1}{a \cosh (\xi)+b \sinh (\xi)}
$$

where $k, \omega, a$, and $b$ are arbitrary constants such that $\omega=k^{3}$, $\xi=k\left(x-\int a(t) d t\right)+\omega\left(\int r(t) d t+C_{1}\right)+\xi_{0}$

$$
u_{20}(x, t)= \pm \frac{k i(b \cosh (\xi)-a \sinh (\xi))}{2(a \cosh (\xi)+b \sinh (\xi))}
$$

where $k, \omega, a$, and $b$ are arbitrary constants such that $\omega=$ $-2 k^{3}, \xi=k\left(x-\int a(t) d t\right)+\omega\left(\int r(t) d t+C_{1}\right)+\xi_{0}$.

\section{Summary and Conclusions}

In summary, motivated by [27], we establish a transform from VC-mKdV equation to the constant coefficient $m K d V$ equation firstly. Then we employ the first integral method and the extended hyperbolic function method to uniformly construct a series of explicit exact solutions for VC-mKdV equations. Abundant explicit exact solutions to VC-mKdV equations are obtained through an exhaustive analysis and discussion for different parameters. The exact solutions obtained in this paper include that of the solitary wave solutions of kink-type, singular traveling wave solutions, periodic wave solutions of triangle functions, Jacobi elliptic function doubly periodic solutions, Weierstrass elliptic function doubly periodic wave solutions, and so forth. In particular, the six explicit exact Jacobi elliptic function doubly periodic solutions $R_{9}-R_{14}$ and Weierstrass elliptic function doubly periodic wave solution $R_{8}$ are uniformly obtained. Some known results of previous references are enriched greatly. The results indicate that the first integral method and extended hyperbolic function method are very effective methods to solve nonlinear differential equation. The methods also are readily applicable to a large variety of other nonlinear evolution equations in physical mathematics.

\section{Acknowledgments}

This work is supported by the NSF of China (40890150, 40890153), the Science and Technology Program (2008B080701042) of Guangdong Province, and the Natural Science foundation of Guangdong Province (S2012010010121). The authors would like to thank Professor Feng Zhaosheng for his helpful suggestions.

\section{References}

[1] R. Hirota, "Exact solution of the modified Korteweg-de Vries equation for multiple collisions of solitons," Journal of the Physical Society of Japan, vol. 33, no. 5, pp. 364-374, 1972.

[2] K. R. Helfrich, W. K. Melville, and J. W. Miles, "On interfacial solitary waves over slowly varying topography," Journal of Fluid Mechanics, vol. 149, pp. 305-317, 1984. 
[3] H. Ono, "Soliton fission in anharmonic lattices with reflectionless inhomogeneity," Journal of the Physical Society of Japan, vol. 61, no. 12, pp. 4336-4343, 1992.

[4] A. H. Khater, O. H. El-Kalaawy, and D. K. Callebaut, "Bäcklund transformations and exact solutions for Alfvén solitons in a relativistic electron-positron plasma," Physica Scripta, vol. 58, no. 6 , pp. 545-548, 1998.

[5] R. K. Dodd, J. C. Eilbeck, J. D. Gibbon, and H. C. Morris, Solitons and Nonlinear Waves, Academic Press, London, UK, 1982.

[6] V. Ziegler, J. Dinkel, C. Setzer, and K. E. Lonngren, "On the propagation of nonlinear solitary waves in a distributed Schottky barrier diode transmission line," Chaos, Solitons and Fractals, vol. 12, no. 9, pp. 1719-1728, 2001.

[7] S. K. Liu, Z. T. Fu, S. D. Liu, and Q. Zhao, "Solution by using the Jacobi elliptic function expansion method for variablecoefficient nonlinear equations," Acta Physica Sinica, vol. 51, no. 9, pp. 1923-1926, 2002.

[8] Z. Yan, "Symmetry reductions and soliton-like solutions for the variable coefficient MKdV equations," Communications in Nonlinear Science \& Numerical Simulation, vol. 4, no. 4, pp. 284288, 1999.

[9] D.-S. Li, Z.-S. Yu, and H.-Q. Zhang, "New soliton-like solutions to variable coefficients MKdV equation," Communications in Theoretical Physics, vol. 42, no. 5, pp. 649-654, 2004.

[10] J. L. Zhang, M. L. Wang, and Y. M. Wang, "An extended Fexpansion method and exact solutions to the KdV and $\mathrm{mKdV}$ equations with variable coefficients," Acta Mathematica Scientia A, vol. 26, no. 3, pp. 353-360, 2006.

[11] C. Dai, J. Zhu, and J. Zhang, "New exact solutions to the $\mathrm{mKdV}$ equation with variable coefficients," Chaos, Solitons \& Fractals, vol. 27, no. 4, pp. 881-886, 2006.

[12] H. Triki and A.-M. Wazwaz, "Sub-ODE method and soliton solutions for the variable-coefficient mKdV equation," Applied Mathematics and Computation, vol. 214, no. 2, pp. 370-373, 2009.

[13] B. Hong, "New Jacobi elliptic functions solutions for the variable-coefficient MKdV equation," Applied Mathematics and Computation, vol. 215, no. 8, pp. 2908-2913, 2009.

[14] Y. Zhang, S. Lai, J. Yin, and Y. Wu, "The application of the auxiliary equation technique to a generalized $\mathrm{mKdV}$ equation with variable coefficients," Journal of Computational and Applied Mathematics, vol. 223, no. 1, pp. 75-85, 2009.

[15] A. H. Salas, "Exact solutions to $\mathrm{mKdV}$ equation with variable coefficients," Applied Mathematics and Computation, vol. 216, no. 10, pp. 2792-2798, 2010.

[16] S. Guo and Y. Zhou, "Auxiliary equation method for the $\mathrm{mKdV}$ equation with variable coefficients," Applied Mathematics and Computation, vol. 217, no. 4, pp. 1476-1483, 2010.

[17] O. Vaneeva, "Lie symmetries and exact solutions of variable coefficient mKdV equations: an equivalence based approach," Communications in Nonlinear Science and Numerical Simulation, vol. 17, no. 2, pp. 611-618, 2012.

[18] X. Hu and Y. Chen, "A direct procedure on the integrability of nonisospectral and variable-coefficient MKdV equation," Journal of Nonlinear Mathematical Physics, vol. 19, no. 1, pp. 1776-1785, 2012.

[19] Z. Feng, "The first-integral method to study the BurgersKorteweg-de Vries equation," Journal of Physics A, vol. 35, no. 2, pp. 343-349, 2002.

[20] Z. Feng, "On explicit exact solutions to the compound BurgersKdV equation," Physics Letters A, vol. 293, no. 1-2, pp. 57-66, 2002.
[21] Z. Feng, "Exact solution to an approximate sine-Gordon equation in $(n+1)$-dimensional space," Physics Letters A, vol. 302, no. 2-3, pp. 64-76, 2002.

[22] Z. Feng, "The first-integral method to the two-dimensional Burgers-Korteweg-de Vries equation," Physics Letters A, vol. 302, pp. 57-66, 2002.

[23] Z. Feng and R. Knobel, "Traveling waves to a BurgersKorteweg-de Vries-type equation with higher-order nonlinearities," Journal of Mathematical Analysis and Applications, vol. 328, no. 2, pp. 1435-1450, 2007.

[24] Z. Feng and G. Chen, "Traveling wave solutions in parametric forms for a diffusion model with a nonlinear rate of growth," Discrete and Continuous Dynamical Systems A, vol. 24, no. 3, pp. 763-780, 2009.

[25] K. Hosseini, R. Ansari, and P. Gholamin, "Exact solutions of some nonlinear systems of partial differential equations by using the first integral method," Journal of Mathematical Analysis and Applications, vol. 387, no. 2, pp. 807-814, 2012.

[26] Y. Huang, Y. Shang, and W. Yuan, "Symbolic computation and the extended hyperbolic function method for constructing exact traveling solutions of nonlinear PDEs," Journal of Applied Mathematics, vol. 2012, Article ID 716719, 19 pages, 2012.

[27] J. He and Y. Li, "Designable integrability of the variable coefficient nonlinear Schrödinger equations," Studies in Applied Mathematics, vol. 126, no. 1, pp. 1-15, 2011. 


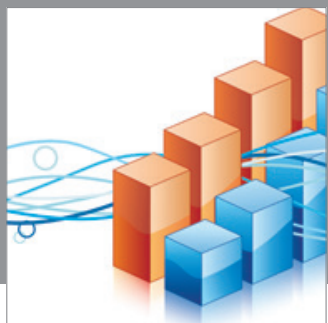

Advances in

Operations Research

mansans

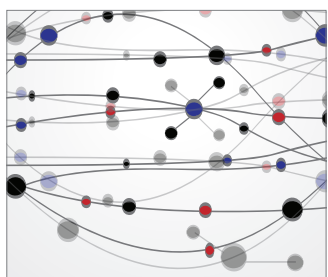

The Scientific World Journal
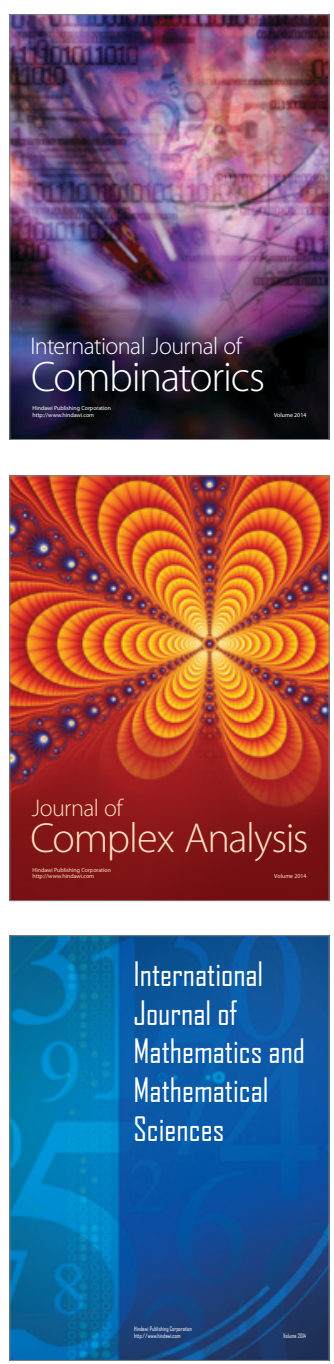
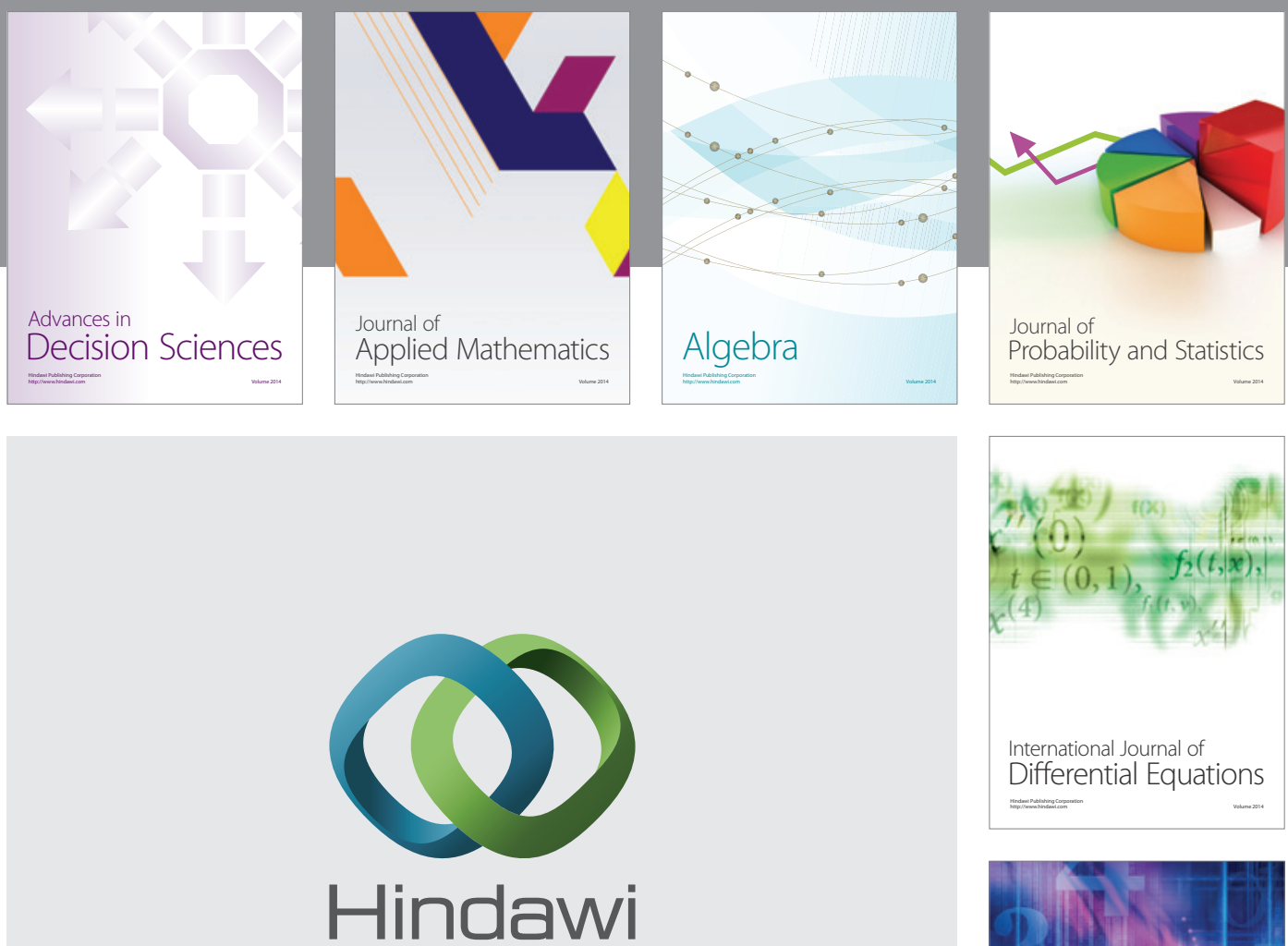

Submit your manuscripts at http://www.hindawi.com
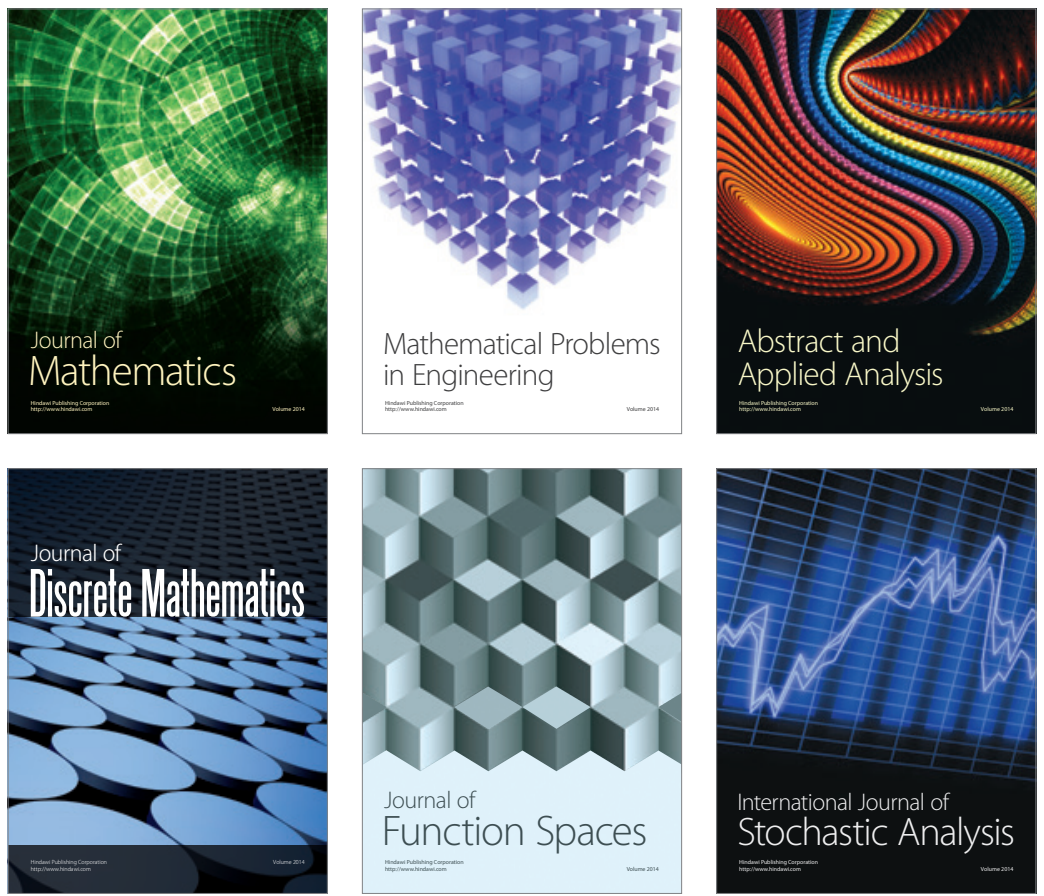

Journal of

Function Spaces

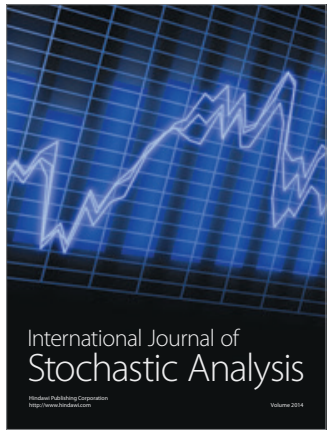

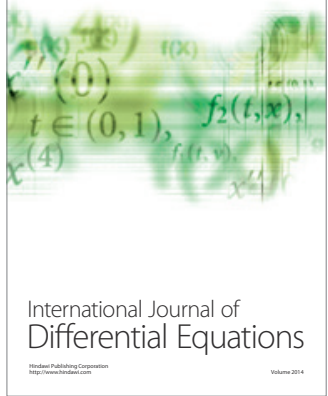
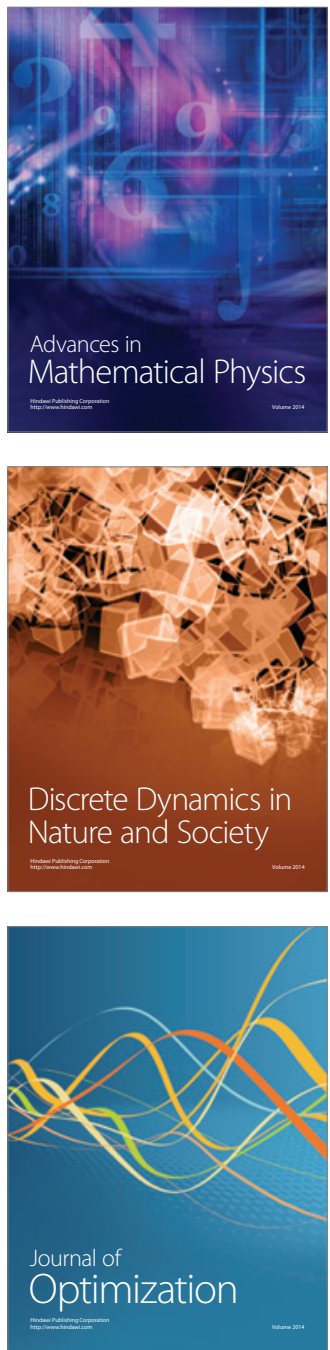